\title{
Transient variations in responding to Pavlovian conditioned stimuli have implications for the mechanisms of "priming"
}

\author{
PENN L. PFAUTZ and ALLAN R. WAGNER \\ Yale University, New Haven, Connectıcut 06520
}

\begin{abstract}
Conditioned responding to a well-trained conditioned stimulus (CS) was assessed in an eyelid conditioning situation under three conditions of prior stimulation: when preceded by recent presentation of the same CS, when preceded by recent presentation of a different CS, and when preceded by no recent stimulation. As compared to the latter condition, prior presentation of the same CS depressed responding, while prior presentation of a different CS augmented responding. Both effects diminished as the interval separating the test stimulus from the preceding CS was lengthened over the range of 2.5 to $10 \mathrm{sec}$. The results were discussed in terms of the interaction of short-term memory representations with subsequent stimulation, and a specific theory concerning the effects of "priming."
\end{abstract}

Much of Pavlov's (e.g., 1927) theory of conditioning was based upon investigations of the transient aftereffects of different training episodes. Thus, he proposed a system, involving the irradiation and concentration of excitation and inhibition, largely to account for the manner in which experience with one conditioned stimulus (CS) would temporarily influence the subsequent responding to other, independently trained CSs. In contrast to Pavlov's extensive work on such phenomena, Wagner, Rudy, and Whitlow (1973) observed that there is almost no modern literature on the short-term performance changes following different Pavlovian training trials and little theoretical concern with how such might be related to long-term memory. Wagner et al. concluded that "it appears that the rejection of Pavlov's theory, which ran counter to accepted neurophysiological views, also carried a depreciation of the data which the theory was constructed to cover (see Loucks, 1933) and an ignoring of the problem area" (1973, p. 424).

The present experiment was designed to evaluate the transient aftereffects of exposure to one CS upon the subsequent responding to that same $\mathrm{CS}$ as opposed to a different but identically trained CS, in the context of rabbit eyelid conditioning. Potential difference in the transient aftereffects in the two cases was viewed as relevant to a recent theoretical formulation offered by Wagner (1976) which suggested that when an event is prerepresented ("primed") in short term memory (STM), further corresponding stimulation may be rendered less effective than it

This research was supported by National Science Foundation Grant BMS 74-20521. Requests for reprints should be addressed to Allan R. Wagner, Department of Psychology, Yale University, New Haven, Connecticut 06520. otherwise would be. Thus, it might be anticipated that responding to the second, or target, CS would be uniquely depressed when recently preceded by exposure to the same, as compared to a different, CS. The results confirm this prediction while revealing other systematic influences that lead us to elaborate on Wagner's (1976) formulation. In a more general vein, the study points to the potential value of further attention to this relatively ignored province of Pavlovian research.

\section{METHOD}

Subjects

The subjects were eight male New Zealand albino rabbits weighing $2-3 \mathrm{~kg}$ at the start of the experiment. Each was housed individually with free access to food and water. One subject died during the course of the experiment, leaving seven from whom data are reported.

\section{Apparatus}

During experimental sessions, each subject was restrained in a $51 \times 18 \times 14 \mathrm{~cm}$ Plexiglas box with only its head protruding. Restraining boxes were enclosed inside individual isolation chambers $(66 \times 48 \times 48 \mathrm{~cm})$ lined with aluminum foil to provide a uniform visual surround and dimly illuminated by a $15-W$ neon bulb. White noise was presented through a $12.7-\mathrm{cm}$ speaker on the rear wall of the chamber so as to maintain a 67-dB sound pressure level (General Radio meter, A scale, re $20 \mu \mathrm{N} / \mathrm{m}^{2}$ ).

Three conditioned stimuli were employed in the experiment, each $1,100 \mathrm{msec}$ in duration. They were a $3,500-\mathrm{Hz}$ tone, a $15-\mathrm{Hz}$ flashing light, and a $60-\mathrm{Hz}$ vibrotactile stimulus. The tone was presented through the same speaker as the white noise and raised the sound pressure level at the locus of the animal's head by $10 \mathrm{~dB}$. The light was generated by a Knight KG-323 strobe lamp mounted behind the rabbit so as to reflect off the walls of the chamber. The vibrotactile stimulus was provided by a Valmour Model 880 hand-massager mounted on the floor of the restraining box in firm contact with the rabbit's chest.

The unconditioned stimulus (UCS) was a $100-\mathrm{msec}$ train of 
$100 / \mathrm{sec}, 4.8-\mathrm{mA}$ square-wave shock pulses delivered through two stainless steel electrodes (Sklar surgical wire, $32 \mathrm{ga}$ ) sutured about the orbit of the subject's right eye. One electrode was implanted approximately $5 \mathrm{~mm}$ below the extreme nasal extent, the other approximately $5 \mathrm{~mm}$ above the extreme lateral extent of the eye. When presented, the UCS overlapped the last $100 \mathrm{msec}$ of the conditioned stimuli.

Closures of the subject's right outer eyelid were monitored by a microtorque rotary potentiometer taped to the rabbit's shaved head and communicating with the eyelid by a length of thread attached to a permanent suture loop. Movements of the eyelid were recorded on a Beckman Dynograph with an effective gain of 4 . A conditioned response was defined as a graphic deflection of $2 \mathrm{~mm}$ (.5 mm of eyelid closure) or more, 140 to $1,000 \mathrm{msec}$ after CS onset.

\section{Procedure}

Pretraining. The purpose of pretraining and the identical retraining sessions between tests was to establish and maintain in each subject levels of responding to each of the three CSs that would potentially permit detection of incremental or decremental effects of the priming manipulation. Each session consisted of 108 trials (36 with each CS) on a reinforcement schedule, which was the same for all cues but was different for different subjects at different stages of training. Trials followed a pseudorandom sequence which equated first-order transitional probabilities of different trial types such that the reinforced or nonreinforced occurrence of a given $\mathrm{CS}$ gave no information about which CS would occur on the next trial or whether or not it would be reinforced. Intertrial intervals ranged from 90 to $150 \mathrm{sec}$ with a mean of $120 \mathrm{sec}$.

All subjects began the experiment with nine sessions on a $50 \%$ reinforcement schedule. Thereafter, the schedules of individual subjects in a given session were increased to $75 \%$ or decreased to $25 \%$ or $0 \%$, as necessary, in an attempt to maintain overall response probability for each subject between $60 \%$ and $70 \%$. This adjustment procedure was likewise employed in subsequent retraining sessions.

Testing. Test sessions were composed of an initial series of 24 "warm-up" trials, 8 with each CS, followed by 24 test units to be described. For four subjects, a $50 \%$ reinforcement schedule was employed during each test session. For the remaining three subjects that tended toward $100 \%$ responding in reinforced sessions, each test session was in extinction.

In any test session, one of the three CSs was selected as the test stımulus, the responding to which was to be evaluated. Test trials were then arranged in which that stimulus was shortly preceded by presentation of the same CS, one of the alternative CSs, or no stimulus. In an attempt to minimize variation in responding due to other local sequencing effects, and to equate the history of reinforcement of the two CSs that could precede the target CS at any point in testing, test units were constructed as depicted in Figure 1. Each test unit included three trials, one with each of the three CSs reinforced or nonreinforced according to the individual subject's schedule of reinforcement. The first trial in the unit was designed as a buffer trial, involving a stimulus, $\mathrm{CS}_{\mathrm{A}}$, not employed as a priming stimulus in that session. The second trial in the unit was the critical measurement trial, with the target stimulus, $\mathrm{CS}_{\mathrm{B}}$, consistently presented $240 \mathrm{sec}$ after the buffer trial. The last trial in the unit involved the remaining stimulus, $\mathrm{CS}_{\mathrm{C}}$, consistently presented $120 \mathrm{sec}$ after the $C_{B}$ trial and $120 \mathrm{sec}$ before the next test unit. The three types of test units, destgnated as Null, Same, or Different, were distınguished by the stimulation that was interpolated between the $\mathrm{CS}_{\mathrm{A}}$ and $\mathrm{CS}_{\mathrm{B}}$ trials In a Null unit, no stimulation was presented. In a Same unit. an isolated "priming" presentation of $\mathrm{CS}_{\mathrm{B}}$ was arranged shortly preceding the $C_{B}$ trial in a Different unit, a similar isolated presentation of $\mathrm{CS}_{C}$ was arranged shortly preceding the $C S_{B}$ trial Fach test session contaned four

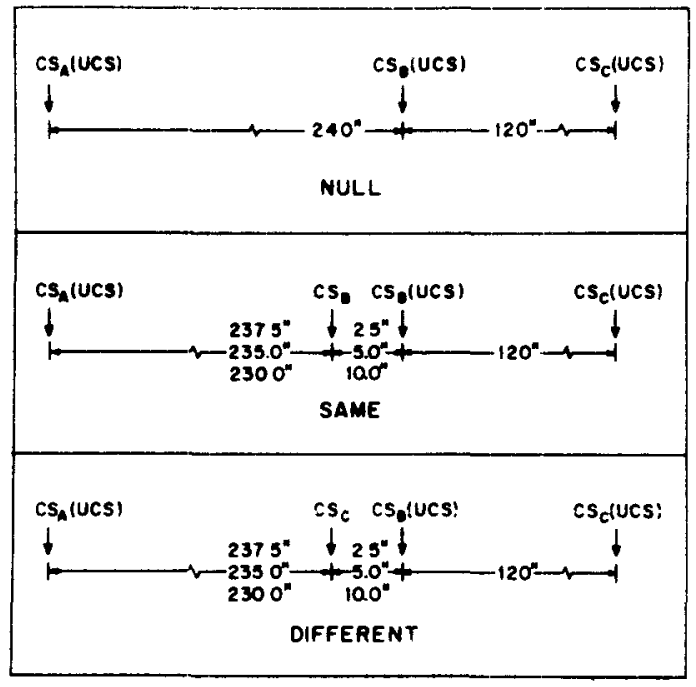

Figure 1. Schematic representation of the three types of test units, each involving a sequence of three trials with three separate $\mathrm{CSs}, \mathrm{CS}_{\mathrm{A}}, \mathrm{CS}_{\mathrm{B}}$, and $\mathrm{CS}_{\mathrm{C}}$, separated by the time intervals (in seconds) indicated. In each unit, the critical test occasion is the trial including the target stimulus, $\mathrm{CS}_{\mathrm{B}}$. In the Null unit, no stimulation shortly precedes the test trial. In the Same and Different units, a CS presentation shortly precedes the test trial, involving the same or a different $\mathrm{CS}$, respectively, as the target. See text for further explanation.

six-unit blocks of two Null, two Same, and two Different units in one of several counterbalanced orders.

The interval separating the priming CS and the target stimulus, like the identification of the target stimulus, was held constant within any test session at $2.5,5.0$, or $10.0 \mathrm{sec}$. In total, 18 separate test sessions were conducted to include every combination of (a) which of the three CSs was identified as $\mathrm{CS}_{\mathrm{B}}$, (b) which of the remaining two $C S s$ was identified as $\mathrm{CS}_{C}$ vs. $\mathrm{CS}_{\mathrm{A}}$, and (c) which of the three test intervals was employed. The sequence of test sessions followed different orders for different subjects. However. in any session, for each number of subjects that had a given pair of cues identified as $C_{B}$ and $C S_{C}$. respectively, there was an approximately equal number of subjects for whom the identification was reversed. Furthermore, the orders of testing were restricted such that in each successive block of six test sessions there were two sessions involving each of the three priming-test intervals.

Prior to testing, the subjects recelved 72 pretraining sessions, of which 8 included a number of test units, as described, but with priming-target intervals of 2.5, 5.0, 10.0, 30.0, and $120.0 \mathrm{sec}$ in relatively unsystematic order between and within sessions. The inclusion of the latter units was an aid to judging the effective intervals to be more carefully explored. The final 18-session test series, from which data are reported, was begun with Session 73, with each successive test session separated from the previous session by $2-8$ days of retraining.

\section{RESULTS}

Percentage responding was successfully maintained at nonextreme values so as to allow the detection of modulating effects of priming. Overall percentage responding to the target CS in the Null units was 55.7 in the 2.5 -sec sessions, 61.3 in the 5.0 -sec sessions, and 59.1 in the $10.0-\mathrm{sec}$ sessions. These values were not reliably different, $F(2,14)<1$. 
Figure 2 summarizes the major results of interest in terms of the mean change in percentage responding to the target CS in the Same and Different test units as compared to the Null units in the test sessions with each priming-test interval. It is apparent that when the target CS was preceded by a different CS there was a facilitation in responding which was more pronounced the shorter the interval separating the stimuli. In contrast, it may be observed that when the target CS was preceded by the same CS there was a depression in responding which was also more pronounced the shorter the interval separating the stimuli.

An analysis of variance performed on the difference scores presented in Figure 2 yielded a reliable main effect of Condition (Same vs. Different) $[F(1,6)=17.27, p<.01]$ and a reliable interaction of Condition and Interval $[\mathrm{F}(2,12)=4.20$, $\mathrm{p}<.05]$. All other effects failed to reach conventional levels of significance: For the main effect of Target Cue, $F(2,12)=2.66, p>.10$; for the Condition by Target Cue interaction, $F(2,12)=$ $2.64, p>.10$; for the main effect of interval, the Interval by Target Cue interaction, and the Condition by Interval by Target Cue interaction, Fs $<1$.

Subsequent t comparisons (two-tailed), using as an error estimate the pooled Subjects by Conditions and Subjects by Conditions by Intervals error terms, revealed that the change in response from the Null condition in the Different condition was reliably greater than zero at 2.5 but not at 5.0 or $10.0 \mathrm{sec}$ $[\mathrm{t}(18)=2.68, \mathrm{p}<.05, \mathrm{t}(18)=1.63, \mathrm{p}>.10$, and $t(18)=1.38, p>.10$, respectively]. The change in responding in the Same condition was reliably different from zero at 2.5 and 5.0 but not at $10.0 \mathrm{sec}[\mathrm{t}(18)=-4.37, \mathrm{p}<.01, \mathrm{t}(18)=-2.89$, $\mathrm{p}<.01$, and $\mathrm{t}(18)=-1.99, \mathrm{p}>.05$, respectively].

\section{DISCUSSION}

There are two major findings of the present study. First, there was a transient impairment of conditioned responding on the occasions of the target CS when the target was recently preceded by presentation of the same stimulus. Second, there was a transient facilitation of conditioned responding on the occasions of the target CS when the target was recently preceded by presentation of a different, but identically trained, stimulus. The former decremental effect is congruent with Wagner's (1976) formulation concerning the effects of "priming" of STM. The latter incremental effect, in this context, however, begs further specification of the mechanism assumed to be involved.

The essence of the priming notion is simply the assumption that when an event is already represented in STM, further corresponding stimulation will be

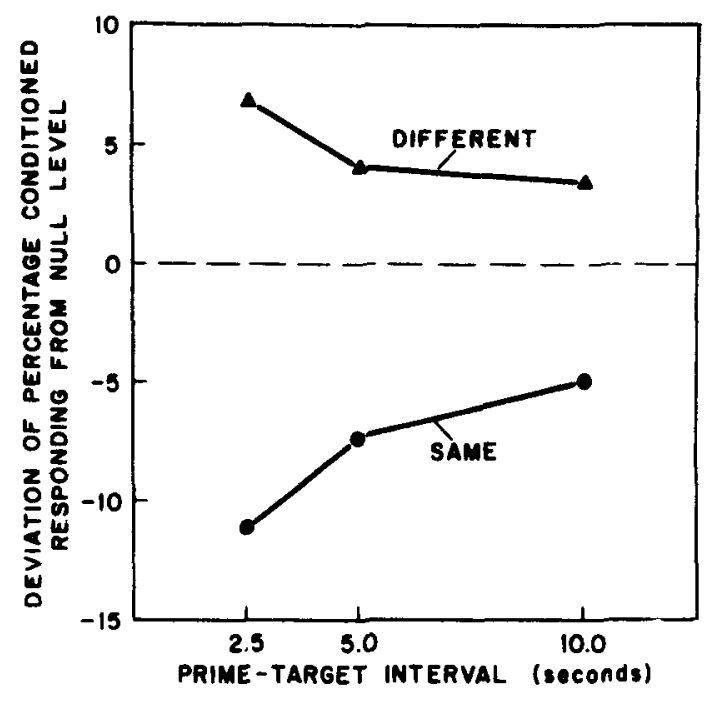

Figure 2. Mean response to the target $\mathrm{CS}$, in the Same and Different test units, presented as the difference in percentage conditioned responding from that in the Null units in the same test sessions. Connected points are from separate sessions with different intervals separating the priming and target stimuli in the Same and Different units.

rendered less effective than it otherwise would be (Wagner, 1976). The most obvious evidence for such an assumption is to be seen in the short-term, refractory-like decrement in responding during a habituation sequence (e.g., Davis, 1970), particularly as such decrement may be shown to be stimulus specific and to be disrupted by other stimulation that would presumably act to clear the effective prerepresentation from STM (Whitlow, 1975). The decremental effects observed in the present study when the target CS was recently preceded by an instance of the same CS may be taken to witness a similar phenomenon. The notable difference is that, in this case, the decrement was observed in a conditioned rather than an unconditioned response to the stimulus. Otherwise, it was likewise shown to be stimulus specific and to dissipate with time between the priming and target stimulus, in a manner consistent with assumptions concerning the transient representation of the priming stimulus in STM.

Wagner's formulation becomes more complex as it allows not only for such instances of response decrement when the priming and target stimuli are the same, but for similar decrements when the priming stimulus is a cue which has otherwise been "associated" with the target stimulus. Thus, it is assumed that a target stimulus may be prerepresented in STM, not only by the prior occurrence of that stimulus (so-called self-generated priming), but also by the occurrence of any other stimulus which acts to retrieve a representation of the target from long-term memory (LTM) (so-called retrievalgenerated priming). Once in STM, the influence of a 
representation is assumed to be indifferent to how it was generated and to act consistently to render corresponding stimulation less effective. Evidence for retrieval-generated priming may be seen in the "conditioned diminution of the UR" in Pavlovian conditioning (e.g., Kimmel, 1966). Likewise, Wagner (1976) has presented evidence to suggest that the long-term response decrement in a habituation sequence [as it may be separated from the shortterm refractory-like effect (e.g., Davis, 1970)] may be attributed to retrieval-generated priming with contextual cues playing the same retrieval role as an explicit CS. Furthermore, if we allow that a prerepiesented stimulus is not only less likely to be immediately responded to, but to be more generally diminished in its effectiveness, evidence for retrievalgenerated priming may also be seen in the diminished reinforcement value (e.g., Kamin, 1969; Rescorla \& Wagner, 1972; Wagner, 1969), diminished retrograde interference effects (Wagner, Rudy, \& Whitlow, 1973), and diminished discriminativetrace effects (Terry \& Wagner, 1975) of a signaled as compared to an unsignaled UCS.

In this context, the facilitative effect observed when the target was preceded by a different CS has substantial implications. Specifically, it suggests that, while prerepresentation of an event in STM may diminish the effectiveness of corresponding external stimulation (as has been assumed), it does not similarly diminish the likelihood or effectiveness of further corresponding representation retrieved from LTM. This distinction requires some elaboration.

It has been assumed above and elsewhere (e.g., Wagner, 1976) that CS presentation results in a perseverating representation of that CS in STM. It has also been assumed that the CS, by virtue of prior pairing with a UCS, will act as a retrieval cue to cause representation of the UCS to be transiently registered in STM, with the occurrence of a conditioned response being the most obvious concommitant of the retrieval process. Now, when CS presentation potentiates the responding observed on the shortly foilowing occasion of a different CS, we are encouraged to assume that the UCS representation retrieved on the occasion of the first CS temporarily persists so that UCS representation adequate to generate a conditioned response is better assured on the occasion of the second CS, granted the usual retrieval action of the latter cue. What does not appear to be reflected is any tendency for UCS representations entered into STM by action of the first CS to interfere with subsequent retrieval of UCS representation from LTM by the second CS.

Figure 3 presents a schematic diagram of the implied mechanism of priming, following a general

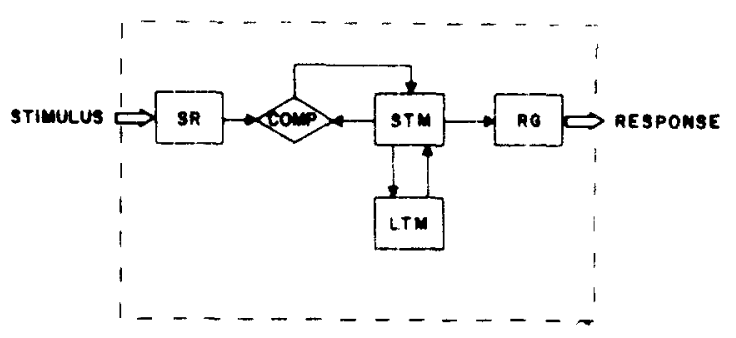

Figure 3. An information processing schema incorporating the assumed mechanism of priming. Information flow between observed stimulus and response is assumed to involve a sensory register (SR), comparator (COMP), short-term memory (STM), long-term memory (LTM), and response generator (RG). See text.

format familiar in information processing models (e.g., Atkinson \& Shiffrin, 1968). The novel assumption included in this diagram is the interposition of a comparator between the sensory register and short-term memory. The relevant flow of information is conceived as follows. Environmental stimulation results in a representation in the sensory register which may or may not have influence upon STM. The comparator is assumed faithfully to examine the contents of the sensory register and the limitedcapacity STM, and to act to transmit to STM only that information in the sensory register which is discrepant from that already in STM. It is this stage of assumed information processing that is calculated to produce the decremental effects of self-generated or retrieval-generated priming: If a stimulus is prerepresented in STM, as a result of either recent exposure to that stimulus or retrieval from LTM, the representation in STM will not be refreshed by exposure to the corresponding stimulus. What should be apparent, however, is that, according to this schema, a representation in STM is not similarly prohibited from being refreshed by retrieval from LTM. Thus, in the relevant example, if one CS has entered STM and promoted retrieval of UCS representation from LTM into STM, a second CS, different from the first, will have its representation transmitted to STM without impediment, where it may further promote retrieval of UCS representation from LTM.

While our conception of the priming process can be explicated with the aid of a diagram such as that in Figure 3, it is important to recognize that the essential notions involved could be represented in the terms of alternative formalisms. In the tradition of Pavlovian conditioning, one might thus be more comfortable with the characterization in Figure 4 which is drawn in likeness to the schemata of Konorski (1967) to emphasize the architecture of memory representations, as opposed to the temporal course of the representations stressed in Figure 3. Following Konorski, we represent in the boxes labeled " $\mathrm{CS}_{\mathrm{A}}$," " $\mathrm{CS}_{\mathrm{B}}$ " and "UCS" func- 
tional units that may enter into associative relations. In Konorski's terminology, they are "gnostic units" that are distinguishable from more "sensory" units (the unlabeled boxes), just as STM and LTM in Figure 3 must be distinguished from the sensory register. The gnostic units in Figure 4 have been joined by dashed arrows to indicate associative linkages, as would presumably result from Pavlovian training, i.e., the UCS unit is presumed to be "excitable" by activity in the $\mathrm{CS}_{\wedge}$ or $\mathrm{CS}_{\mathrm{B}}$ units as well as by presentation of the UCS. The existence of the gnostic units with such associative linkages corresponds to LTM, while STM would be identified with the set of currently active gnostic units. Thus, to speak in the language of Figure 3 of stimulus representation in STM would be equivalent to speaking of transient activation of a particular gnostic unit, while retrieval from LTM would be equivalent to such activation via an assocrative linkage.

In this schema, the priming mechanism would take the form of an inhibitory feedback route from each gnostic unit to its own sensory input channel, as indicated in Figure 4. ${ }^{1}$ Thus, for example, transient activation of the UCS unit would make that unit temporarily less susceptible to further activation from corresponding sensory input, and it should do so whether the initial activation was a result of UCS presentation (self-generated priming) or of CS presentation (retrieval-generated priming). At the same time, activation of the UCS unit per se would not make that unit less susceptible to further activation via associative linkages. Thus, transient activation of the UCS unit via C $^{\prime}$, would not inhibit further activation via $\mathrm{CS}_{n}$.

The schema of Figure 4, incorporating the assumption that unit activity perseverates beyond the occasions of stimulation and involves an inhıbitory feedback mechanism consistent with Wagner's (1976) formulation, can obviously allow for the incremental and decremental effects of a priming stimulus as observed in the present study. To be applied to many other situations relevant to Wagner's general formulation, it would need to be made considerably more detailed. For example, the assumption of a limited-capacity STM would need to be accommodated, perhaps in the manner that Konorski (1967) has suggested, via lateral inhibition between units within the same "gnostic field." And decisions would have to be made concerning the associative architecture involved in extinction and the behavioral indexes of "inhibition" (e.g., Wagner \& Rescorla, 1972). As Pavlov (1927) saw, such specification may be aided by research on the transient aftereffects of different training episodes as well as on the relatively permanent products of conditioning.

As a final postscript, we must acknowledge an interpretive possibility that has thus far been

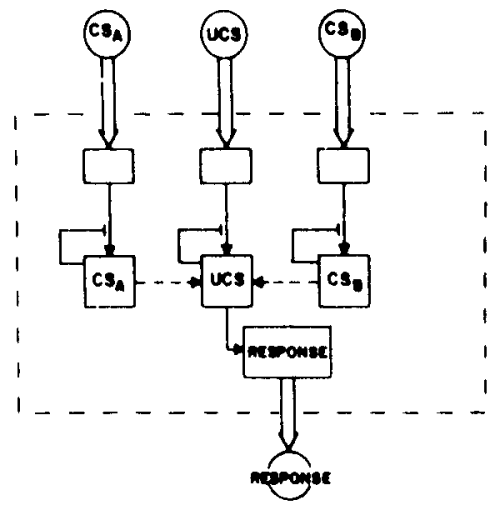

Figure 4. Alternative schematization of assumed mechanism of priming emphasizing the architecture of memory representation. Between the observed stimuli and response there is assumed to occur activation of various units as a result of the interaction of stable "excitatory" connections (solid arrows), acquired "excitatory" connections (broken arrows), and stable "inhibitory" connections (stopped lines). The labeled units are the "gnostic units" among which acquired connections are presumed to be formed. See text.

ignored. Perhaps the decremental effects seen in this study were due to the fact that the priming CS was nonreinforced and thus produced some extinction of the CS-UCS association, detectable when the subsequent target CS was the same but not when it was different. This view is made less attractive by the fact that the decrement decayed over the brief priming-target intervals employed and was accompanied by a similarly decaying incremental effect when the CSs were different. Yet, it is impossible completely to discount such possibility, particularly if one is willing to entertain some additional principle of associative forgetting such as that embodied in Estes' (e.g., 1959) stimulus fluctuation model. At this juncture, we can only look toward the results of additional research to comment further upon our assumption that CS representation in STM renders corresponding stimulation generally less effective, beyond any extinction of specific associations.

\section{REFERENCES}

Atkinson, R. C., \& Shiffrin, R. M. Human memory: A proposed system and its control processes. In K. W. Spence \& J. T. Spence (Eds.). The psuchology of learning and motivation (Vol. 2). New York: Academic Press, 1968.

Davis. M. Effects of interstimulus interval length and variability on startle-response habituation in the rat. Journal of Comparative and Physiological Psychology, 1970, 72. 177-192.

Estes, W. K. The statistical approach to learning theory. In S. Koch (Ed.), Psychology A study of a science (Vol. 2). New York: McGraw-Hill, 1959

Kamin, L. J. Predictability, surprise, attention and conditionıng. In B. Campbell \& R. Church (Eds.). Punishment and aversive bihavior. New York: Appleton-Century-Crofts. 1969.

Kimmel. H. D. Inhibition of the unconditioned response in "lassical cond itoming. Psvchological Rev'lew. 1966, 73, 232-240 
KonoRSKI, I Integrative activity of the brain. Chicago. University of Chicago Press. 196\%.

Loucxs. R. B An apprassal of Pavlov's systematization of behavior from the experimental standpoint Journal of Comparatue Psychologv. 1933, 15, 1.47.

PAviov. I. P. Conditioned reflexes. London: Oxford University Press, 1927.

Rescorla. R. A., \& Wagner, A. R. A theory of Pavlovian conditioning: Variations in the effectiveness of reinforcement and nonreinforcement. In A. H. Black \& W. F. Prokasy (Eds.). Classical condtitioning II Current theory and research. New York: Appleton-Century-Crofts, 1972.

TerRy. W. S., \& WAGNer, A. R. Short-term memory for "surprising" versus "expected" unconditioned stimuli in Pavlovian conditioning. Journal of Experimental Psychology. Animal Behavior Processes, 1975, 1, 122-133.

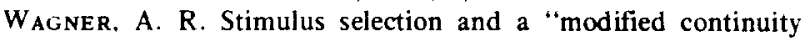
theory". In G. H. Bower \& J. T. Spence (Eds.), The psychology of learning and motivation (Vol. 3). New York: Academic Press, 1969.

WAGNer, A. R. Priming in STM: An information processing mechanism for self-generated or retneval-generated depression in performance. In T. J. Tighe \& R. N. Leaton (Eds.), Habutuation. Perspectives from child development, animal behavior, and neurophystology. Hillsdale, N.J. Lawrence Erlbaum Associates. 1976.
W AGNeR, A. R., \& Rescorla, R. A. Inhibition in Pavlovian condition ing: Application of a theory. In R A. Boakes \& M. S. Halliday (Eds.), Inhibitton and learning. New York. Academic Press, 1972.

W AGNER, A. R., RuDY, J. W., \& WhitLow, J. W. Rehearsal in anmal conditioning. Journal of Experimental Psychology, 1973, 97, 407-426. (Monograph)

Whitrow, J. W. Short-term memory in habituation and dishabituation. Joumal of Experimental Psychology: Animal Behavior Processes, 1975, 1. 189-206.

\section{NOTE}

1. This inhibitory feedback route bears superficial resemblance to Konorski's (1967) "inhibitory perceptive recurrent reflex." We are cautious in suggesting more substantial similarity, in that Konorski called upon this reflex only in regard to a limited set of phenomena (habituation) and then offered a picture of the reflex route (via a nonspecific arousal system) that would not necessarily be well suited to our general account of priming.

(Received for publication July 24,1975 ; revision accepted November 11, 1975.) 